\title{
Genome sequencing in healthcare: understanding the UK general public's views and implications for clinical practice
}

\author{
Lisa M. Ballard $\mathbb{D}^{1} \cdot$ Rachel H. Horton ${ }^{1} \cdot$ Angela Fenwick $^{1}$ Anneke M. Lucassen $\mathbb{D}^{1}$
}

Received: 24 May 2019 / Revised: 6 August 2019 / Accepted: 22 August 2019 / Published online: 16 September 2019

(c) The Author(s), under exclusive licence to European Society of Human Genetics 2019

\begin{abstract}
Technological advances have seen the offer of genome sequencing becoming part of mainstream medical practice. Research has elicited patient and health professional views on the ethical issues genome sequencing raises, however, we know little about the general public's views. These views offer an insight into people's faith in such technologies, informing discussion regarding the approach to consent in clinic. We aimed to garner public views regarding genome sequencing, incidental findings (IFs), and sharing genetic information with relatives. Participants $(n=1954)$ from the British general public completed a survey, distributed via email. Overall, the public had a positive view of genomic sequencing, choosing 'informative' as the most popular word (52\%) and 'family legacy' as the most popular analogy (33\%) representing genomic sequencing for them. Fifty-three percent agree that their relative had the right to be told about genetic information relevant to them. Fifty-four percent would expect to be told about IFs whether they had asked for them or not. Clinical practice needs to acknowledge these perspectives and expectations in order to facilitate meaningful discussion during the consent process for genomic tests. We suggest that: (a) optimistic perspectives on the usefulness of genomic tests need to be tempered by discussion in clinic about the likelihood that genomic results might be uninformative, uncertain or unexpected; (b) discussions regarding the familial nature of results are needed before testing: the majority of patients will welcome this and any concerns can be explored further; and (c) a wider discussion is required regarding the consent approach for genomic testing.
\end{abstract}

\section{Introduction}

Genomic testing in healthcare is transitioning from being accessible mainly via specific initiatives such as the 100,000 Genomes Project, to becoming widely available as a standard test in the UK healthcare system [1]. The Global Alliance for Genomics and Health (GA4GH) anticipates that over sixty million people worldwide will have had their genome sequenced in a healthcare context by 2025 [2]. The rise of direct-to-consumer genetic testing companies means

Supplementary information The online version of this article (https:// doi.org/10.1038/s41431-019-0504-4) contains supplementary material, which is available to authorized users.

$\triangle$ Lisa M. Ballard

1.ballard@soton.ac.uk

1 Clinical Ethics and Law at Southampton (CELS), Centre for Cancer Immunology, University of Southampton, School of Medicine, Southampton, UK that genomic information is also increasingly accessible outside of formal healthcare settings [3]. However, the ways in which the general public think of genomic tests-the majority of whom will have had no exposure to genetic medicine - and their responses to the ethical issues raised by such testing, have been relatively underexplored [4].

How genomics is talked about in popular culture means that it is likely that many people considering genomic testing will be approaching consent conversations with pre-existing views about what sort of information they may receive from genomic tests. Gaining knowledge regarding patient expectations in reference to genomic tests may enhance the consent approach in the clinical context. Public perception is becoming more positive about what genomics might offer over time, which may be related to the discourse on genomics presenting information derived from genetic and genomic tests in a positive and deterministic light [5]. Mainstream media articles often focus on the excitement and promise of 'personalised medicine' [6], and direct-to-consumer companies have financial incentives to present genetic and genomic 
tests as universally useful and accurate [7]. However, clinical interpretation of genomic data is often uncertain or significantly context-dependent, and the process by which clinically meaningful results are distilled from raw genomic data is complex and sometimes opaque [8].

Genomic tests can reveal information that has relevance not only to that individual but also to family members [9]. Professional guidance alerts clinicians to the fact that a result in one person may mean that others need to be told about their risks. This will usually happen via the patients, or with their consent, but sometimes clinicians will need to consider breaching the confidence of one person in order to warn another (or find a way of doing so that does not breach individual clinical confidences) [10]. Current research suggests that many patients are happy with this approach and see their genetic information as belonging to their family as well as themselves [11]. In contrast, clinicians are often worried about protecting individual confidentiality [12], and struggle with a concern that, by providing general information about a family risk they might enable someone to correctly infer medical information about a specific person in their family, and thereby breach that person's confidentiality.

Genomic tests may reveal clinically relevant unexpected information (incidental findings (IFs)), that is a result that was not sought or was unrelated to the reason for having testing (also called unsolicited or secondary findings, but we will use IFs in this case as a broad term [13]). There has been much debate over recent years on what should be reported routinely, communicated to patients, or what choice patients should have in these matters. As shown previously, patients have mixed views about receiving information about IFs [14], however, those taking part in research have shown a preference towards receiving a wide range of possible results generated from genome sequencing [15].

Given that more work is required to better understand the views and opinions of the general population in regard to these issues [4], we designed a survey to assess the opinions of a representative sample of the adult population living in the UK. Our survey explored views and opinions regarding genomic tests in general; genomic information with familial implications and how this should be shared; and whether and how IFs should be communicated. We consider these views in the context of existing research on patient views and discuss the implications for UK clinical practice.

\section{Method}

The study protocol was approved by the University of Southampton, Faculty of Medicine Ethics Committee.

\section{Participants}

An online survey was conducted in August 2018 via YouGov, a company who has access to a community of 6 million people worldwide [16]. YouGov was chosen based on past experience of working with them to successfully undertake a survey regarding genetic testing of children for adult-onset conditions [17]. YouGov sent an email with a link to our survey to a sub-population $(n=2005)$ of their UK community of over 1 million respondents. The subsample was representative of the population of Great Britain (GB) in terms of age, gender, social class and education. YouGov incentivises their participants by giving them 50 points for each survey they complete, and points can either be accumulated and exchanged for money ( $£ 50$ payment upon reaching 5000 points) or entered into a monthly prize draw to win more points or cash prizes.

\section{Questionnaire}

The questions aimed to ascertain how people conceptualise genomic tests in healthcare, in particular what they think regarding the potential of such tests to reveal unexpected information, or information of relevance to relatives of the person being tested. The questions were developed in discussion by experts in questionnaire design, genetic medicine, social science, ethics and piloted with six members of a Patient and Public Involvement group (myKinMatters Research Partners). Experts in questionnaire design aided in the phrasing of questions and answers to ensure we would get useful data and research partners checked the clarity of the questions and made suggestions for other answers they would like to see. The survey (see Supplementary information) began with an opening statement explaining that the questions would be about genome sequencing in a healthcare context and a sensitivity screener was used to give responders the option of not continuing with the survey questions. The questions were presented in a fixed order. For questions 4-7, possible answers were presented in a random order to each participant (with 'prefer not to say' and 'don't know' at the end). Sociodemographic data were collected by YouGov on respondents' gender, age, socioeconomic status and place of residence. YouGov did not collect data on the average time it took respondents to complete the survey; however, we piloted our survey using Survey Monkey with our six research partners which reported an average of $5 \mathrm{~min}$ and $16 \mathrm{~s}$ to complete.

\section{Data analysis}

All analyses were weighted such that the balance of sociodemographic characteristics of the sample population reflected that of the GB adult population. Analyses were 
performed using the IBM Statistical Package for the Social Sciences v24. Descriptive statistics were used to conduct the majority of the analyses, and missing data were excluded for each question. Socioeconomic status categories A, $\mathrm{B}$ and $\mathrm{C} 1$ were grouped together, as were $\mathrm{C} 2, \mathrm{D}$ and $\mathrm{E}$.

\section{Results}

\section{Survey respondents}

YouGov $^{1}$ circulated the survey to 2005 people and 1954 $(97.4 \%)$ indicated - after being advised of the topic-they were happy to complete it. We were informed that based on other surveys that YouGov had administered with a sensitivity screener (topics included cancer, bereavement and mental health), between $95-99 \%$ of respondents continue with the survey. Characteristics of people invited to take the survey are detailed in Table 1.

\section{Perception of genome sequencing in healthcare}

Respondents were given a range of words and asked to choose the three that they most associated with genome sequencing. The options included positive words (predictive, informative, certain, helpful and reliable), negative words (scary, messy, problematic, unclear and worrying) and neutral words (personal and context-dependent). Most people chose positive and neutral words to describe genome sequencing (Fig. 1), with the three most selected words being 'informative' (52\%), 'personal' (37\%) and 'helpful' $(30 \%)$. The most chosen negative word was 'worrying' (16\%).

The most frequent combination was two positive words and a neutral word, as shown in Fig. 2. The least frequent was three negative words.

When asked to choose which of various phrases best represented how they thought about genome sequencing in healthcare, positive analogies such as 'family legacy' and 'your instruction manual' were the most popular (Table 2). 'A needle in a haystack' was the least popular analogy (3\%). Twenty-one percent of people did not select an answer (i.e. selected prefer not to say, don't know, or none of these).

\section{Sharing genetic information within families}

Most respondents agreed that relatives of a person with a genetic condition have a right to be told if they might also

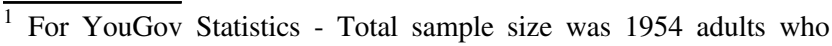
agreed to take part in the survey. Fieldwork was undertaken between 28th - 29th August 2018. The survey was carried out online. The figures have been weighted and are representative of all GB adults (aged 18+).
}

Table 1 Characteristics of people who completed the survey $(n=$ 1954)

\begin{tabular}{llc}
\hline Characteristics & & $n(\%)$ \\
\hline Gender & Female & $1016(52)$ \\
& Male & $938(48)$ \\
Age & $18-24$ & $216(11.1)$ \\
& $25-34$ & $281(14.4)$ \\
& $35-44$ & $351(18)$ \\
& $45-54$ & $329(16.8)$ \\
Social grade & 55+ & $776(39.7)$ \\
& A, B, C1 & $1121(57.4)$ \\
Working & C2, D, E & $833(42.6)$ \\
& Full time & $776(38.7)$ \\
& Part time & $339(16.9)$ \\
& Student & $108(5.4)$ \\
& Retired & $502(25)$ \\
& Unemployed & $78(3.9)$ \\
& Not working/other & $202(10.1)$ \\
\hline
\end{tabular}

$\overline{\mathrm{a}} \mathrm{AB}$ - higher and intermediate managerial, administrative, professional occupations

C1-supervisory, clerical and junior managerial, administrative, professional occupations

C2-skilled manual occupations

DE-semi-skilled and unskilled manual occupations, unemployed and lowest grade occupations

have inherited it (89\%) (Table 3). In one question we asked respondents to imagine themselves as having a genetic condition that their relatives might also have inherited but not know about; in another we asked respondents to imagine their relative being diagnosed with a genetic condition that might have relevance for them. In both cases, more than half of respondents strongly agreed that relatives have a right to be told if a genetic diagnosis is made in a family member that might have relevance for their health $(53 \%$ and $58 \%$ respectively). Interestingly, just over $4 \%$ of respondents to both questions selected 'tend to disagree' or 'strongly disagree' to having a right to be told.

Two-thirds $(68 \%)$ of respondents reported that they would want to tell relatives themselves if they were found to have a genetic condition that was also relevant to their relatives. One third of respondents would want help to do this from their health professional (HP), and the other third would not (Table 4). Over half (56\%) of respondents reported that if they themselves were the one to receive genetic health information from their relative, they would prefer their relative to tell them (Fig. 3). Only 2\% $(n=37)$ of respondents did not want their relatives informed under any circumstances if a result found in them was relevant to their family members, and only $2.6 \%(n=51)$ did not want their relatives to share a genetic result with them. 
Fig. 1 Words respondents most associated with genome sequencing (respondents were instructed to select three words, however, some only selected one or two)
Fig. 2 Combination of negative, positive, and neutral words chosen ('positive' words given score of +1 ; 'neutral' words given score of 0 ; 'negative' words given score of -1 )
Table 2 The phrase respondents felt most closely represented how they thought about genome sequencing (respondents were instructed to select one answer)

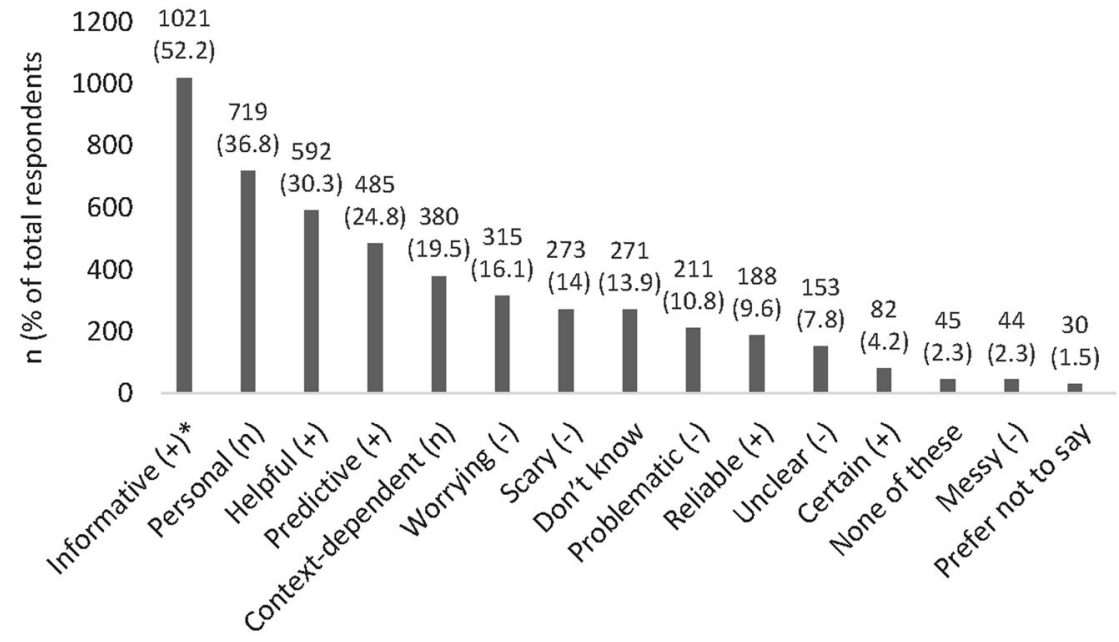

Words

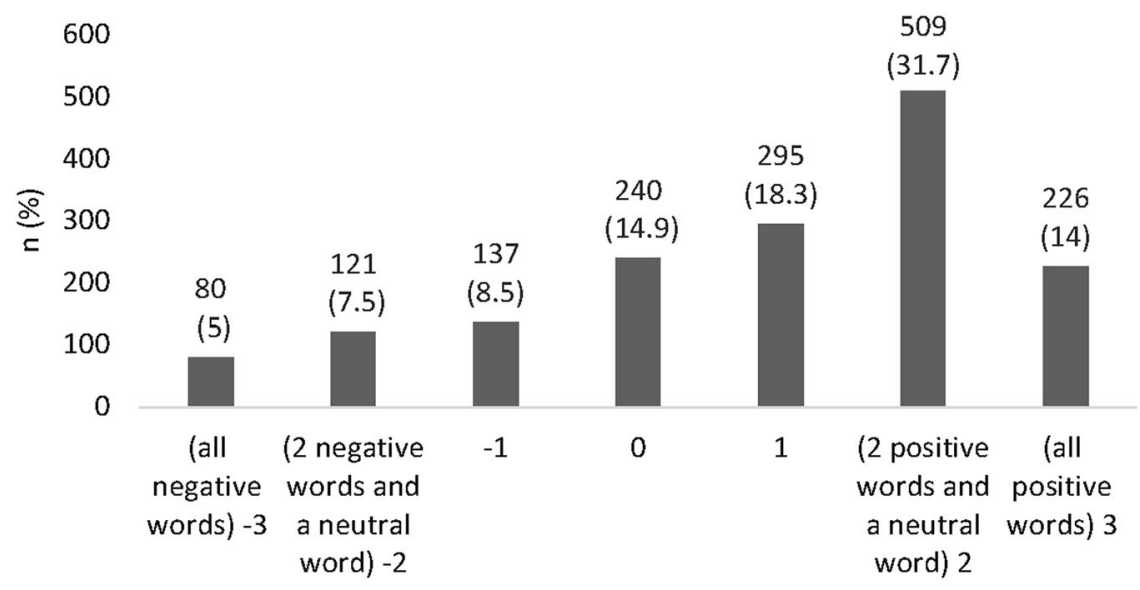

Score for all three words

\begin{tabular}{ll}
\hline Phrase & $n(\%)$ \\
\hline Family legacy—information to help me and my family to understand our health & $640(32.7)$ \\
'Your instruction manual'—-helping you understand your own health better & $371(19)$ \\
Don't know & $308(15.8)$ \\
Pandora's box—unlocking secrets which it might be better to leave undisturbed & $197(10.1)$ \\
An encyclopaedia or Wikipedia—a resource for you to obtain information as and when you & $145(7.4)$ \\
need it. & $137(7)$ \\
Fortune-telling—revelations that may be good, bad, or may never happen & $78(4)$ \\
None of these & $50(2.5)$ \\
'A needle in a haystack'—so unlikely to find anything that will influence how I manage my & \\
health. & $28(1.4)$ \\
Prefer not to say &
\end{tabular}

\section{Incidental findings}

Over half $(54 \%)$ of all respondents expected to be told about IFs, irrespective of whether they had asked for them (Fig. 4). A quarter (25\%) wanted to know about IFs for which there was a high chance of developing the condition, and one fifth (20\%) wanted to know if they were warned about the possibility of IFs before-hand; 
only $3 \%$ of respondents did not want to be told about them.

Further analysis can be found in Supplementary information.

\section{Discussion}

Our survey explored respondents' views regarding genome sequencing, in particular its capacity to generate unexpected information (IFs), and information with familial relevance. Most people will not have had personal experience of genomic testing, yet $97 \%$ of people invited to take our survey were willing to answer questions about it after reading the sensitivity screener. Many expressed strong views (i.e. selected strongly agree) in response to those questions, suggesting that people engaging with genomic testing may come to the process with pre-existing views and expectations as to what genome sequencing might and should offer. The results of this survey can inform clinical

Table 3 Answers to two questions about respondents and their relatives' right to know about genetic information found in their family

\begin{tabular}{llc}
\hline Question & Answer & $n(\%)$ \\
\hline If I find out I have a genetic & Strongly agree & $1033(52.9)$ \\
condition and that my relatives & Tend to agree & $706(36.1)$ \\
might also have inherited it but do & Tend to disagree & $61(3.1)$ \\
not know about this possibility, & Strongly disagree & $26(1.3)$ \\
they have a right to be told. & Don't know & $115(5.9)$ \\
& Prefer not to say & $13(0.7)$ \\
If my relative finds out they have a & Strongly agree & $1125(57.6)$ \\
genetic condition that I might also & Tend to agree & $614(31.4)$ \\
have inherited but not know about, & Tend to disagree & $62(3.2)$ \\
I have a right to be told. & Strongly disagree & $21(1.1)$ \\
& Don't know & $109(5.6)$ \\
& Prefer not to say & $23(1.2)$ \\
\hline
\end{tabular}

practice by indicating common attitudes to information ascertained via genome sequencing.

\section{People expect that genome sequencing in healthcare will be helpful}

Respondents to our survey indicated a positive and potentially deterministic view of genome sequencing, describing it as 'informative', 'personal' and 'helpful'. Only $5 \%$ of people chose exclusively negative words to describe genome sequencing. Similarly, the most popular analogies for genome sequencing in healthcare positioned it as a useful enterprise: a 'family legacy' or 'instruction manual' (33\% and $19 \%$ respectively). Seventy-nine percent of people were prepared to select an analogy to represent how they thought about genome sequencing in healthcare, although most had probably not had genome sequencing, they identified with particular ways of conceptualising it. It is worth noting, however, that the word 'worrying' was the sixth most commonly chosen word (16\%), suggesting some ambivalence regarding genome sequencing.

Our findings complement existing research: Ipsos MORI garnered views from the public regarding what genomics adds to the social contract and found that the majority of respondents had positive views and high expectations of genomics in healthcare [18]. In addition, a study that interviewed research participants having non-diagnostic genome sequencing found that many patients expected to learn information about their genetic makeup that would allow them to plan and prepare for the future. Many participants also suggested that results would give them an absolute answer on whether they would develop a particular disease [19].

Popular optimism around the clinical worth of genome sequencing likely reflects the positive discourse around genomics and 'personalised medicine' in the mainstream media [20]. It may also result from exposure to advertising from direct-to-consumer genetic testing companies, which have commercial incentives to present information
Table 4 What respondents would like to happen if they had their genetic code tested and a genetic condition was found that was also relevant to their relatives [respondents were instructed to select one answer]

\begin{tabular}{lc}
\hline Answer & $n(\%)$ \\
\hline I would want to tell my relatives without help of a health professional & $679(34.8)$ \\
I would want my health professional to help me tell my relatives & $652(33.4)$ \\
I would want the health professional to tell my relatives, if I don't tell them as they have a duty & $173(8.9)$ \\
of care to do so & $169(8.6)$ \\
Don't know & $137(7)$ \\
I would want my health professional to tell my relatives for me & $72(3.7)$ \\
I would want another member of my family/a friend to tell my relatives & $37(1.9)$ \\
Not applicable-I would not want my relatives to be told under any circumstances & $24(1.2)$ \\
Prefer not to say & $11(0.6)$ \\
Other &
\end{tabular}


Fig. 3 Ways in which respondents would most like their relative to share their genetic test results with them [respondents were instructed to select the response they MOST preferred]
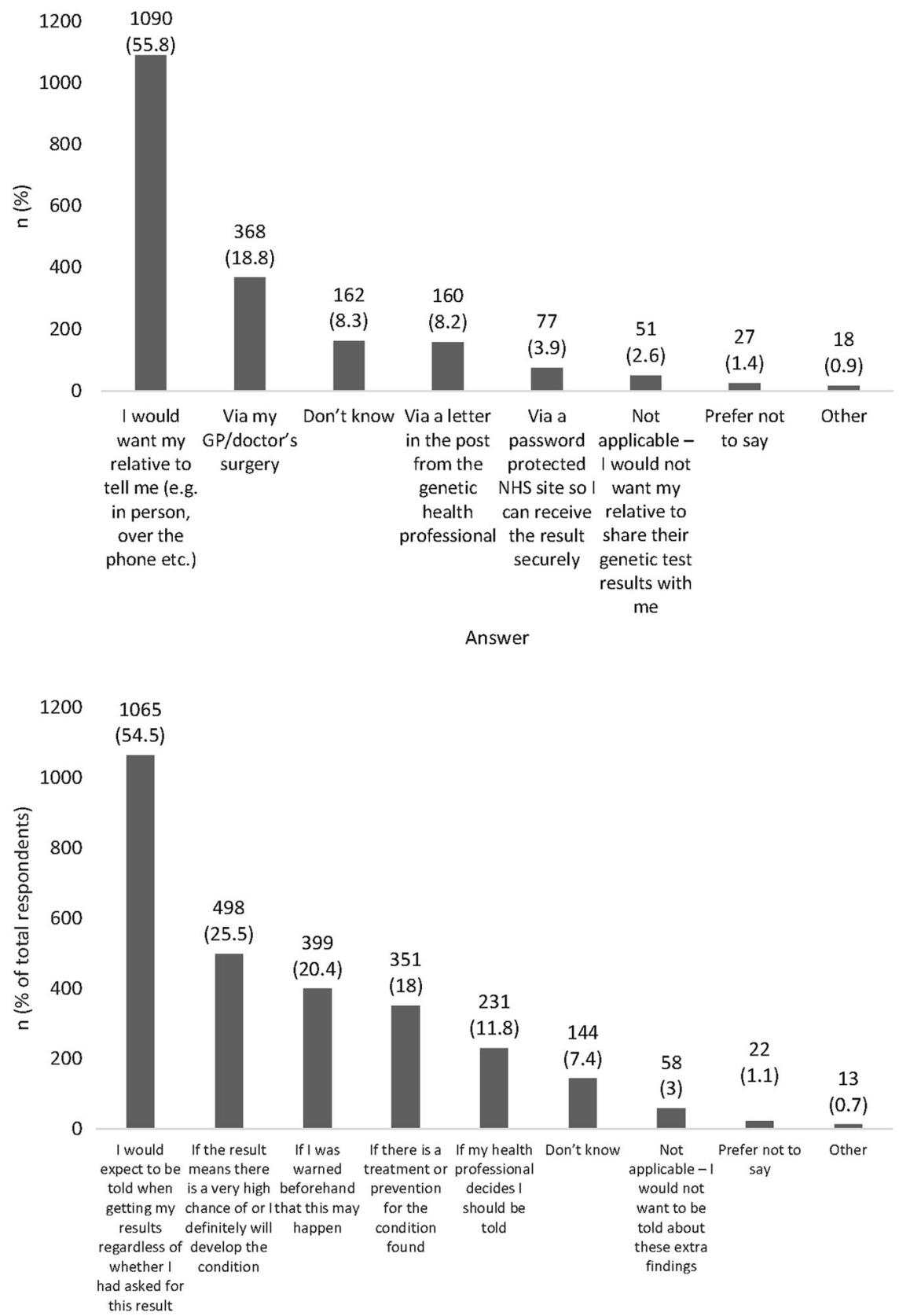

Answer from genomic testing as valuable and useful [21]. An enthusiastic but somewhat reductive view of genomics permeates health policy at the highest levels, as illustrated by recent discussions about the value of Matt Hancock's (current UK Health Minister) polygenic risk score indicating a higher lifetime chance of prostate cancer [21]. His polygenic risk score indicating a $15 \%$ risk of prostate cancer by age 75 was hyped as life-saving ('This test may have saved my life'), even though it did not represent a significant departure from population risk (lifetime risk of $18 \%$ ), and more men die with, rather than from, prostate cancer [22].
Whilst genome sequencing is leading to significant health benefits and at times changing clinical care for the better [23], a wholly positive view of genome sequencing arguably sets people up for disappointment and disillusion and could ultimately result in the loss of public trust in genomics and the NHS in general [18]. Over half of genomic tests do not find a clear answer for the problem that led to a person having the test, at least in the short term [24]. Variants identified via genomic tests may be challenging to interpret, and their predictive value is often much reduced if found outside the context of a family history of the relevant disease [25]. This mismatch between the expectation and 
realities of genomic testing can be potentially harmful, and the difficulties involved are an increasingly frequent theme at Genethics (a UK wide forum to discuss and explore difficult ethical issues encountered in genomic medicine www.ethox.ox.ac.uk/ethics-support/genethics-club).

We argue that in light of our survey results, it may be important that clinicians emphasise the potential limitations of genome sequencing when discussing genomic tests with patients. The evidence that many people will be positively disposed towards genome sequencing and expect it to be instructive, means that much of the work of selling the benefits of genomic testing may already have been done before a patient comes to clinic. This emphasises the importance of finding out what a patient might be expecting from genome sequencing in advance of arranging the test. If a person starts with a disproportionately optimistic view of what genome sequencing might lead to, then in order to facilitate choice, and to 'inform' consent as far as practicable, clinicians may need to dedicate more time to explaining potential pitfalls and limitations of genome sequencing. This also suggests a need for more tempered discussions around genomics in schools and in the popular discourse.

\section{Genomic information as family information}

Our survey found that most respondents thought that they had a right to know if a genetic condition was found in their relative that might be relevant to them (and vice versa). This indicates that many people think of their genomic information as familial; indeed, 'family legacy' was the most popular analogy for conceptualising genome sequencing in healthcare. This aligns with existing research regarding the views of patients having genetic tests - they did not feel that knowledge derived via this process would be 'mine and nobody else's'[11]. It is interesting to note that most respondents were happy that this sharing of results might happen in either direction; broadly, they indicated that they were as willing to share their own result as they were to receive a relative's.

Our findings inform a challenging area of practice. Professional guidance has increasingly taken the stance that genetic information should be viewed as confidential to families, not individuals (though the personal consequences for individuals of having a genetic variant should be confidential to them) [26]. However, clinicians often struggle to apply this concept in practice, expressing concerns that such an approach might put strain on family relationships, or undermine patient trust in the NHS [12]. Whether an individual has a 'right of veto' over communicating genetic information of potential relevance to their family is currently being tested in the courts in the ABC vs St George's case regarding the ethical—and possibly legal—issue of
HPs duty to warn relatives of familial genetic risks of disease [27].

The potential for genome sequencing to find information of familial relevance makes consent challenging. Testing one person might by implication test other members of their family, however consent processes often position the person providing a DNA sample as the sole decision-maker. The Chief Medical Officer's Generation Genome report highlighted this difficulty, suggesting the need for a renegotiation of the social contract for genomics in the NHS to reflect that genomic information may inherently stray outside the boundaries of a conventional one-to-one interaction between a patient and clinician [28]. Previous research has shown that the public have ambivalent views regarding the sharing of genomic results within families and HPs role in this [18].

Our survey adds to evidence that in the context of heritable genetic risk many people may be more comfortable with a familial approach to genomic information than HPs might expect. However, we highlight the need to discuss implications for relatives during the consent process for genomic testing. Whilst the majority of respondents felt that they had a right to know if a genetic condition was diagnosed in someone else in their family that might be relevant for them (and vice versa), over 4\% disagreed. Here, it would be important to explore why they disagreed, and to discuss what might happen if they were found to have a genetic diagnosis that might be relevant to their relatives, in order to help them make an informed decision about testing. From a practical perspective, respondents also held a variety of views as to how they would want to share or receive information if genome sequencing in one person revealed a familial risk.

Our results underline the benefit of discussing such issues in advance of a person having genome sequencing, emphasising that many people engaging in genomic testing will not be starting as a 'blank slate' with no pre-existing views on this topic. Our survey suggests that many people might welcome the opportunity to discuss strategies for sharing information. Of the respondents who would want to tell their relatives about a genetic condition, $49 \%$ would want help from an HP, raising the question of how this might work in practice. Our research group is currently developing a web application and online intervention (myKinMatters), which HPs could direct their patients to for support with informing relatives [29]. Only $3.1 \%$ of people either did not want their relatives told or preferred not to answer the question.

We also call into question to what extent it is helpful to focus on the 'right not to know', a challenging concept since a person needs to know there is something to know to be able to act on that right. The potential for infringing a 'right not to know' is given much prominence in the 
bioethical literature [30], but only $2.6 \%$ of respondents to our survey suggested that they would not want a relative to share their genetic test results with them. Clearly, the rarity with which people indicate a wish to preserve a right not to know, does not justify ignoring this issue. However, it does suggest that other issues in the sharing of genomic information deserve at least comparable research focus. For example, for the majority of people who do express a theoretical intention to share and receive genomic information: how can we facilitate and support this, so that information is delivered in a sensitive, accurate, and timely manner?

\section{Many people would expect to be told if unexpected information was incidentally found via genome sequencing}

Our survey found that $54 \%$ of respondents would expect to be told if unexpected health-relevant information was incidentally found in the course of genomic testing, regardless of whether they had asked for this. This aligns with existing research showing that many people express an interest in receiving a wide range of results from genomic data [15]. One study which garnered public views from 75 countries, including the UK, found that this enthusiasm extended even to the level of raw sequence data from genomic research: $61 \%$ of respondents surveyed were interested in receiving this, and using it to seek out further interpretations [31].

Our study goes further in indicating that many people not only want to know if unexpected information is found in the course of genome sequencing, but they would also expect to be told such information even if they had not asked for it. This aligns with research currently in progress exploring the views of 100,000 Genomes Project participants, which found that some participants could not accurately recall whether they had chosen to know 'additional findings' (genetic predispositions to a specified list of diseases, unrelated to the main condition that led to the participant having genome sequencing). Whatever they had indicated on their consent form for the project, most people expressed that they would want and expect to be told if 'anything important' was found [29].

Not accurately recalling decisions calls into question the weight that we place on consent in the process of deciding which information from genome sequencing should be reported as results. Currently, historical consent forms are often deferred to in attempts to resolve dilemmas about whether to communicate IFs from genomic tests. For example, a 100,000 Genomes Project participant's choice to receive 'additional findings' might be used to support the idea that they would also want to know IFs from their test. However, our survey suggests that many people would not expect HPs to rely on consent to 'do all the work'-they might expect to be told about IFs regardless of whether they said this at the time of consenting to their genomic test. We are not of the opinion that our data signals a preference for an opt-out model of consent. We are suggesting that if IFs are found in the course of genomic testing, patients may expect HPs to engage with these findings and disclose them, rather than expect HPs to stick to the remit of the original test and act as if the IFs were never seen. We highlight the need for wider discussion and recognition of this issue-if consent cannot do everything, what else should inform decisions as to whether and how genomic information is communicated?

\section{Limitations}

As for any survey, there was some potential for selection bias, for example all respondents needed to be able to use online systems. We aimed to minimise bias by working with YouGov to weight the data such that different sociodemographic groups were proportionately represented. We did not ask whether respondents had previously had genetic or genomic testing, so were unable to ascertain whether previous experience might have influenced how people answered the questions in our survey. We do know that considerably less than $1 \%$ of the UK population will have had genome sequencing in a healthcare context [2], meaning that less that 20 people in our sample are likely to have undergone genome sequencing.

The use of genome sequencing in healthcare is a complex and challenging subject and many respondents may not have thought about this topic previously. This may also be the case for many patients referred to the new Genomic Medicine Service (all mainstream specialities offering genomic sequencing), and part of the role of HPs is to inform patients about genome sequencing and facilitate decision-making around it. Our survey aimed to elicit the views that people might bring to an initial consultation about genome sequencing, so for these purposes it did not matter that many people taking the survey might not be well informed about the topic.

We asked the survey questions in a set order (i.e. questions about the familial status of genomic information, followed by questions about IFs, followed by questions about how people think of genomic sequencing in healthcare). We asked questions about how people conceptualise genomic sequencing last, because they were more abstract, and we thought it would be easier for people to engage with these questions after they already started to think about genome sequencing in healthcare over the course of the previous questions. However, it is possible that the questions that we previously asked people to consider may have influenced the choices that they ultimately made as to how they conceptualise genomic testing in healthcare overall. The information that we gave about genomic sequencing at 
the start of the survey might also have influenced people's responses (see Supplementary information). We purposely avoided making value statements about the results from such technologies, as we though this might unduly influence respondent's answers.

A further limitation is that whilst this survey expresses respondents' theoretical views, these do not necessarily reflect what they would actually do in practice. For example, research indicates that whilst many patients intend to communicate their genetic results with their relatives [32], many find it difficult to do so in reality [33]. However, whilst we think that this potential discrepancy between theory and practice needs highlighting, it also presents an opportunity. Our data indicates that many people are positively disposed towards sharing or receiving genomic information within a family; this suggests that research as to how we might practically facilitate this has an audience and the potential to make an impact.

\section{Conclusion}

In summary, our survey indicates that many people begin with optimistic perspectives on the usefulness of genomic tests: we think this needs to be tempered by more discussion about the likelihood that genomic results might be uninformative or uncertain, both in popular discourse, but also in the context of patient-clinician conversations about genomic testing. Most respondents thought that they had a right to know if a genetic condition was found in their relative that might be relevant to them (and vice versa). We suggest that this indicates that many people would accept that genetic information can be considered confidential at a familial, as opposed to an individual level, and highlights the need to discuss this during the consent process as we continue to explore what this might look like in practice. Lastly, our results indicate that many people actively expect to be told about IFs from genome sequencing, regardless of whether they have asked for this. We argue that clinical practice needs to take account of these popular perspectives and expectations, in order to facilitate meaningful consent for genomic tests. These findings call into question the extent to which we can rely on consent forms to reflect people's wishes about what sort of information they might expect to receive from genomic tests, and indicates a need to consider more widely what other factors might inform decision-making as to what to include in the 'results' of genome sequencing.

Acknowledgements We wish to thank Gillian Crawford (CELS), Emily Griffiths (YouGov), and Rafaella Metaxa, a student from the University of Southampton MSc in Genomic Medicine, for their assistance with the design of the questionnaire. We would also like to thank Ruth Pickering from the University of Southampton for help with data analysis, Juliette Schuurmans (CELS), Kathy Kendall (CELS), and our PPI group for their help with the construction of questions. This work was supported by funding from a Wellcome Trust collaborative award [grant number 208053/Z/17/Z (to $\mathrm{AL})$ ] and funding from a Wessex Medical Research Innovation Fund award.

\section{Compliance with ethical standards}

Conflict of interest The authors declare that they have no conflict of interest.

Publisher's note Springer Nature remains neutral with regard to jurisdictional claims in published maps and institutional affiliations.

\section{References}

1. Caulfield M, Davies J, Dennys M, Elbahy L, Fowler T, Hill S, et al. The 100,000 genomes project protocol. London: Genomics England; 2015.

2. Birney E, Vamathevan J, Goodhand P. Genomics in healthcare: GA4GH looks to 2022. bioRxiv. 2017.

3. Covolo L, Rubinelli S, Ceretti E, Gelatti U. Internet-based directto-consumer genetic testing: a systematic review. J Med Internet Res. 2015;17:e279.

4. Roberts J, Middleton A. Genetics in the 21st century: implications for patients, consumers and citizens. F1000Res. 2017;6:2020.

5. Henneman L, Vermeulen E, van El CG, Claassen L, Timmermans DR, Cornel MC. Public attitudes towards genetic testing revisited: comparing opinions between 2002 and 2010. Eur J Hum Genet. 2013;21:793-9.

6. Marcon AR, Bieber M, Caulfield T. Representing a "revolution": how the popular press has portrayed personalized medicine. Genet Med. 2018;20:950-956.

7. Hall JA, Gertz R, Amato J, Pagliari C. Transparency of genetic testing services for 'health, wellness and lifestyle': analysis of online prepurchase information for UK consumers. Eur J Hum Genet. 2017;25:908-17.

8. Horton R, Lucassen A, Fenwick A. Unpacking the concept of a genomic result. Am J Bioeth. 2019;19:70-71.

9. Parker M, Lucassen AM. Genetic information: a joint account? $\mathrm{Br}$ Med J. 2004;329:165-7.

10. JCMG. Consent and confidentiality in clinical genetic practice: guidance on genetic testing and sharing genetic information. 2nd ed. London: Royal College of Physicians and Royal College of Pathologists; 2011.

11. Dheensa S, Fenwick A, Lucassen A. 'Is this knowledge mine and nobody else's? I don't feel that.' Patient views about consent, confidentiality and information-sharing in genetic medicine. $\mathrm{J}$ Med Ethics. 2016;42:174-9.

12. Dheensa S, Fenwick A, Lucassen A. Approaching confidentiality at a familial level in genomic medicine: a focus group study with healthcare professionals. BMJ Open. 2017;7:e012443.

13. Shkedi-Rafid S, Dheensa S, Crawford G, Fenwick A, Lucassen A. Defining and managing incidental findings in genetic and genomic practice. J Med Genet. 2014;51:715-23.

14. Clift KE, Halverson CM, Fiksdal AS, Kumbamu A, Sharp RR, McCormick JB. Patients' views on incidental findings from clinical exome sequencing. Appl Transl Genom. 2015;4:38-43.

15. Facio FM, Eidem H, Fisher T, Brooks S, Linn A, Kaphingst KA, et al. Intentions to receive individual results from whole-genome sequencing among participants in the ClinSeq study. Eur J Hum Genet. 2013;21:261-5.

16. YouGov. 2018. https://yougov.co.uk/about/panel-methodology/. 
17. Shkedi-Rafid S, Fenwick A, Dheensa S, Lucassen AM. Genetic testing of children for adult-onset conditions: opinions of the British adult population and implications for clinical practice. Eur J Hum Genet. 2015;23:1281-5.

18. Ipsos MORI. A public dialogue on genomic medicine: time for a new social contract. London: Ipsos MORI; 2019.

19. Hylind R, Smith M, Rasmussen-Torvik L, Aufox S. Great expectations: patient perspectives and anticipated utility of nondiagnostic genomic-sequencing results. J Community Genet. 2017;9:19-26.

20. Marcon AR, Bieber M, Caulfield T. Representing a "revolution": how the popular press has portrayed personalized medicine. Genet Med. 2018;20:950-6.

21. Schaper M, Schicktanz S. Medicine, market and communication: ethical considerations in regard to persuasive communication in direct-to-consumer genetic testing services. BMC Med Ethics. 2018;19:56.

22. BBC. Hancock criticised over DNA test 'over reaction'. BBC; 2019 https://www.bbc.co.uk/news/health-47652060.

23. Horton RH, Lucassen AM. Recent developments in genetic/ genomic medicine. Clin Sci. 2019;133:697-708.

24. Steward CA, Parker APJ, Minassian BA, Sisodiya SM, Frankish A, Harrow J. Genome annotation for clinical genomic diagnostics: strengths and weaknesses. Genome Med. 2017;9:49.

25. Wright CF, West B, Tuke M, Jones SE, Patel K, Laver TW, et al. Assessing the pathogenicity, penetrance, and expressivity of putative disease-causing variants in a population setting. Am J Hum Genet. 2019;104:275-86.
26. Lucassen A, Hall A. Consent and confidentiality in clinical genetic practice: guidance on genetic testing and sharing genetic information. Clin Med. 2012;12:5-6.

27. Gilbar R, Foster C. It's arrived! relational autonomy comes to court: ABC v ST George's Healthcare NHS Trust [2017] EWCA 336. Med Law Rev. 2018;26:125-33.

28. Davies SC. Annual report of the Chief Medical Officer 2016, generation genome. In: Health Do, editor. London: Department of Health; 2017.

29. Ballard LM, Dheensa S, Fenwick A, Lucassen A. The use of an electronic health record to facilitate communication of additional findings in families (P20.06B). Copenhagen: European Society of Human Genetics Conference; 2017.

30. Chadwick R, Levvitt M, Shickle D. The right to know and the right not to know: genetic privacy and responsibility. 2nd ed. In: Chadwick R, Levvitt M, Shickle D, editors. Cambridge: Cambridge University Press; 2014.

31. Middleton A, Wright CF, Morley KI, Bragin E, Firth HV, Hurles $\mathrm{ME}$, et al. Potential research participants support the return of raw sequence data. J Med Genet. 2015;52:571-4.

32. Roshanai AH, Rosenquist R, Lampic C, Nordin K. Does enhanced information at cancer genetic counseling improve counselees' knowledge, risk perception, satisfaction and negotiation of information to at-risk relatives?-a randomized study. Acta Oncol. 2009;48:999-1009.

33. Wiseman M, Dancyger C, Michie S. Communicating genetic risk information within families: a review. Fam Cancer. 2010;9: 691-703. 